\title{
Ideas sobre la minería en Antioquia. Desarrollo, gobernanza comunitaria y arreglos colectivos
}

Ideas About Mining in Antioquia. Development,
Community Governance and Collective Settings

Adolfo Eslava ${ }^{1}$

Profesor titular, Universidad EAFIT, Medellín, Colombia

aeslava@eafit.edu.co

http://orcid.org/0000-0003-2213-0765

Andrés Preciado ${ }^{2}$

Profesor catedrático, Universidad EAFIT, Medellín, Colombia

apreciad@eafit.edu.co

http://orcid.org/0000-0001-8116-0599

Recibido: 19-02-2018

Aprobado: 29-05-19

2 Magíster en Gobierno y Políticas Públicas. 


\section{Resumen}

Este artículo presenta una discusión conceptual en torno a las ideas sobre la minería en Antioquia. A través de un acercamiento cualitativo que mezcla experiencia en campo y análisis conceptual, el artículo parte de las fallas mercantiles y estatales para promover el desarrollo y, por ello, tiene como objetivo presentar la gobernanza comunitaria como categoría analítica que permita comprender a la comunidad, sus atributos y capacidades, en particular, en el contexto de la minería aurífera. En breve, el artículo presenta los elementos analíticos que permiten conectar arreglos cotidianos con procesos de decisiones públicas, esto es, microfundamentar las macrohipótesis para llegar a escenarios en donde las políticas públicas sean el resultado de acción colectiva.

Palabras clave: desarrollo económico; minería; gobierno; política pública.

\section{Abstract}

This article addresses the conceptual discussion around the ideas on the subject of the mining industry in Antioquia. Through a qualitative approach which mixes field experience and conceptual analysis, the article is based on the mercantile and state failures to promote development and thus it aims to introduce community governance as an analytical category which allows understanding the community, its attributes and capabilities, particularly, in the context of gold mining. In short, the article presents the analytical elements that allow the connection between quotidian arrangements and public decision-making processes, namely, microfundament macro-hypotheses to reach scenarios where public policies are the result of collective action.

Keywords: Political economy; Extractivism; Governance; Public policy; Mining.

¿Cómo citar este artículo? / How to quote this article?

Eslava, A. y Preciado, P. (2019). Ideas sobre la minería en Antioquia. Desarrollo, gobernanza comunitaria y arreglos colectivos. Sociedad y economía, (38), 6-20. https://doi.org/10.25100/sye.voi38.6212 
"Sería ideal poder microfundamentar las grandes macrohipótesis"

(González, 2003, p. 21)

\section{Introducción}

Este artículo surge de la necesidad de entender conceptualmente la manera en que se concibe la minería aurífera, en particular, al escenario variopinto de actividades culturales, económicas y políticas que engloba esta denominación, desde los asuntos que aluden al oro como maldición hasta aquellos que permiten afirmar que quien tiene el oro, pone las reglas. Por tanto, la aproximación que se describe en este artículo busca descifrar, a partir de referencias bibliográficas y experiencias de indagación en campo, los usos manidos e ideas sobre la minería de oro en escenarios de exploración y explotación donde confluyen diversos actores provenientes tanto de instancias legales como ilegales, así como de representantes de Estado, empresa y comunidades.

Cabe señalar que la construcción analítica aquí presentada se deriva de la investigación "Gobernanza de la Minería Aurífera en Buriticá", cofinanciada por Proantioquia y adscrita al Grupo de Investigación "Sociedad, Política e Historias Conectadas" (Categoría Al, Colciencias), de la Universidad EAFIT. Se alimenta de la experiencia empírica de trabajos previos como "Oro como Fortuna" e "Informalidad e ilegalidad en la explotación del oro y la madera en Antioquia", del mismo grupo de investigación. Esto implica que el contexto de análisis territorial se circunscribe a Antioquia, en particular a aquellas zonas del departamento con actividad minera: uno de los mayores productores del metal en el país históricamente, de ahí la relevancia de su análisis.

El artículo está dividido en cinco secciones, incluida esta introducción. La segunda sección discute las macrohipótesis sobre la minería del oro y el contexto económico en el que se desarrolla, dominado por una dicotomía entre mercado y Estado. La sección siguiente plantea los microfundamentos necesarios para mejorar el entendimiento de las macrohipótesis y las razones que pueden explicar su ausencia analítica. En la cuarta sección se presenta la idea de la gobernanza comunitaria como la forma de superar la exclusión de las comunidades. Se cierra con las consideraciones finales.

\section{El espacio de las macrohipótesis: superar la dicotomía Estado - mercado incluyendo a la comunidad}

Una de las causas del predominio económico en las ciencias sociales reside en el programa sistemático de investigación que caracteriza a la ciencia económica, desde la cuestión por la riqueza que formuló Adam Smith, en su libro conocido como La Riqueza de las Naciones, hasta el auge del siglo XX, cuando se indagó por el crecimiento económico y sus determinantes. El resultado es que la ciencia económica está presente en nuestra vida cotidiana debido a que existe una ruta histórica que traduce postulados teóricos -bien argumentados y apoyados en evidencia empírica- en criterios internacionales de política pública y, al final, afectan dinámicas no solo económicas, sino también políticas y sociales en los territorios subnacionales.

Por ejemplo, la siguiente afirmación es diciente respecto al alcance que ha tenido la ciencia económica en los terrenos empíricos que afectan nuestra vida cotidiana:

La proposición del libre mercado es un sólido logro intelectual con gran atractivo estético, pero su indiscriminado mal uso ha tenido enormes implicaciones para el mundo, sobre todo, la manera en que construimos las políticas, pensamos acerca de la globalización y descartamos a los detractores (Basu, 2013, p. 11).

Desde una visión clásica de la teoría económica, el desarrollo se ha planteado como el resultado del crecimiento económico en manos del mercado, ayudado por la capacidad del Estado en la prestación de los servicios y el cumplimiento de 
las tareas que le corresponden. De esta manera, la interdependencia entre arreglos mercantiles y estatales se concibe alrededor de una relación causal, según la cual, primero se crece para luego desarrollarse (Eslava, 2016). No obstante, esta forma de entender el desarrollo, bajo un alto grado de predominio económico, pone la discusión en términos de dicotomía entre Estado y mercado, pero desestima tópicos centrales de la relación entre economía y sociedad. En términos de Ostrom (2013):

\begin{abstract}
Este punto de vista dicotómico del mundo explicó los patrones de interacción y resultados relacionados con los mercados para la producción y el intercambio de bienes estrictamente privados, pero no ha logrado explicar de forma adecuada las dinámicas internas dentro de las empresas privadas. Tampoco aborda de forma adecuada la amplia diversidad de acuerdos institucionales que las personas desarrollan para gobernar, proveer y administrar los bienes públicos y los recursos comunes (p. 9).
\end{abstract}

En el caso de la minería de oro, en Antioquia, la idea generalizada en los municipios mineros se ha centrado en la explotación del recurso dorado por parte de ilegales; en otras ocasiones por parte de alguna empresa formal, con un telón de fondo de precariedad estatal que anula la dicotomía en favor del arreglo privado y convierte a los territorios en agentes pasivos de la actividad minera. Así las cosas, los retos de desarrollo no se pueden reducir a la pregunta por cuánto Estado y cuánto mercado requieren los arreglos sociales. En contextos mineros, para atender la complejidad de las variables culturales, económicas y políticas que están en juego, también es necesario incursionar en la vida cotidiana de las comunidades, y en la forma en que resuelven dilemas colectivos y resisten arreglos nocivos.

En consecuencia, el error de la falsa dicotomía entre Estado y mercado se ha centrado en darle mayor relevancia al crecimiento económico y su objeto de estudio clásico, la riqueza, olvidando las dimensiones humana y comunitaria: el comportamiento de las personas, sus preferencias, interacciones y atributos prosociales.
Se hace necesario entonces superar la visión dicotómica entre Estado y mercado, a partir de la inclusión de la comunidad. Así, se hace posible una concepción en la que los tres actores se pueden confrontar, pero también complementar. Ya que el actor comunitario puede entrar al juego con la posibilidad de minimizar costos de transacción y asimetrías de información, de tal forma que adquiere la condición de jugador relevante dentro de la discusión económica y no un espectador más (Bowles y Gintis, 2005). Esta idea se soporta en la necesidad de contrarrestar el predominio de la tenaza estatal mercantil, desde la promoción de la tríada Estado - mercado - comunidad, en interacción, que facilite el contagio mutuo entre sus principios de equidad, eficiencia y reciprocidad, y entre sus fines, alrededor de la distribución de la riqueza, en un ambiente de confianza generalizada.

Esto implica, además, romper con el planteamiento de que los retos o problemas de desarrollo se tramitan acudiendo a mayor intervención del Estado o mayor intervención del mercado, dependiendo del caso. Por el contrario, la inclusión del actor comunidad reivindica la centralidad de los arreglos comunitarios y de otro tipo de arreglos, en muchas ocasiones informales (Guha-Khasnobis, Kanbur y Ostrom, 2006), que existen y se hacen presentes en la interacción entre los tres actores, en un marco en el que los problemas sociales son complejos, multicausales y, por tanto, requieren respuestas más ingeniosas y novedosas, complementarias a las miradas convencionales de asignación eficiente de recursos o medidas asistencialistas.

Con respecto a la minería del oro, en Antioquia, la falta de regulación sobre las condiciones de extracción o el desgobierno minero (Giraldo, 2013) aunado a algunas prácticas empresariales desligadas del contexto comunitario en el que están asentadas y la extracción ilícita del mineral, evidencian la incapacidad del Estado y el mercado para generar desarrollo en los territorios mineros. Los procesos históricos de la minería en Antioquia se han caracterizado por su apego arbitrario a la norma, lo que brinda grandes dimensiones a la minería ilegal ante la mira- 
da pasiva de las autoridades. Recientemente, la normatividad ha asumido la responsabilidad de construir el marco legal requerido. Sin embargo, al decir de los habitantes de territorios mineros, las empresas mineras obtienen la licencia ambiental, pero carecen de licencia social. Asuntos que refuerzan la propuesta de inclusión de las comunidades como forma de superar la falsa dicotomía.

La mirada estrictamente económica es insuficiente para abordar la amplitud del debate en mención. En Colombia, la dimensión del reto es enorme, pues la mayor parte del territorio es un espacio colectivo -títulos a resguardos y a consejos comunitarios, parques nacionales, ecosistemas costeros, aguas continentales y marítimas, espacios públicos urbanos, títulos mineros- que exige estudiar los arreglos cotidianos a los que llegan los pobladores del espacio común, "por ello la importancia de comprender los mecanismos mediante los cuales se logra la acción colectiva necesaria para su mantenimiento" (Cárdenas, 2009, p. 5).

En este punto, es importante destacar que cuando el debate se caracteriza por adjetivos que (des) califican las formas de concebir los modos de producción y el modelo de desarrollo como extractivista (Garay, 2013) o colonial (Suárez, 2013), la contraparte -Estado y gran empresa- reacciona con una defensa que alega alarmismo distante de la realidad. Sin embargo, este tipo de voces e ideas está llamado a enriquecer la discusión; en particular, el componente económico de la reflexión y las decisiones, pueden complementarse con las ideas provenientes del análisis político, ecológico y social. En este sentido, las recomendaciones del experto alemán Hans-Jürgen Burchardt (2014) para avanzar en la fundamentación empírica y analítica del extractivismo son pertinentes para el caso colombiano:

Una de las cuestiones centrales es si seremos capaces de convertir, y en qué medida, el incremento cuantitativo de las rentas en una redistribución cualitativa y en participación social. Otra, en qué medida será posible vincular funcionalmente el trabajo a la productividad, es decir, reducir la economía informal. Y, en especial, cómo tratarán las sociedades extractivas los territorios de su base de reproducción con respecto a la representación, el reconocimiento y la redistribución, pues estos constituyen el núcleo de su existencia (p. 13).

De allí la necesidad de apostar por un marco conceptual metateórico, promotor de la síntesis interdisciplinaria con base en elementos de convergencia para el diálogo de saberes, metodologías y hallazgos con repercusiones en la política pública. El sistema socioecológico (SSE) entendido como constructo societal y ecológico - "dinámica de la construcción/deconstrucción del territorio como proceso societal, cultural y geofísico" (Garay, 2014)- es un acierto analítico de enorme relevancia y oportunidad para el caso de la minería aurífera colombiana. En efecto, desde allí se pueden emprender las comparaciones entre SSE de orden nacional con el fin de brindar más evidencia respecto a nuevas omisiones de la política económica global.

Las conclusiones de este nivel de análisis permiten enriquecer el debate público, respecto a cuestiones centrales sobre las que a menudo se legisla sin suficiente ilustración de los argumentos en contienda: tributación, empleo, inversión extranjera, conflictos sociales. En breve, de la mano de la serie auspiciada por la Contraloría General de la República, el país ya cuenta con un completo panorama de las implicaciones de una minería extractiva.

La socio-ecología política, la gobernanza democrática adaptativa y la gestión societal de la minería como sistema complejo, constituyen un todo integral, en su diversidad y en medio de una multiplicidad de relaciones, retroalimentaciones, impactos, instancias de acción determinante, agentes y grupos sociales, intereses, simbologías, perspectivas temporales, etc., en y entre cada uno de los subsistemas componentes: social y ecológico en sentido amplio (Garay, 2014, p. 6).

Justamente, el SSE permite comprender que la argumentación de índole económica es apenas una de las varias facetas que configura el con- 
texto microsituacional minero. En ese sentido, es necesario complementar la indagación por las condiciones que rodean la actividad minera desde la vida cotidiana del habitante de los lugares de explotación.

Bajo la perspectiva de sistemas socioecológicos, no cabe esperar que la relevancia del tema se alcance por la vía de catástrofes que alteren el sistema de recursos. En su lugar, es preciso incursionar de manera decidida en la promoción de la bandera ambientalista, en el debate político, para alterar el sistema societal de gestión. Por ejemplo, los escenarios electorales son una oportunidad para visibilizar la cuestión ambiental a través de nuevos liderazgos, que logren convertir la conciencia ambiental en contienda electoral.

\section{El terreno de los microfundamentos: arreglos microinstitucionales que informan decisiones públicas}

Como se advirtió en la introducción, este artículo y sus postulados se enmarcan en un programa institucional (en la Universidad EAFIT) de investigación, alrededor de la pregunta por la economía política de la periferia colombiana. A sabiendas de que "la combinación de mejores herramientas metodológicas y una mayor apreciación de la naturaleza compleja y contingente de las relaciones sociales y ecológicas ha disminuido el apoyo a soluciones genéricas y preconcebidas en las políticas públicas" (Poteete, Janssen y Ostrom, 2012, p. 481).

El día de su muerte, aparecía en Project Syndicate la que fuera su última comunicación dentro del amplio legado escrito que dejó Elinor Ostrom. Green from the Grassroots es un texto breve que hace consideraciones valiosas para abordar la cuestión humana. En opinión de la única mujer laureada con el Nobel de Economía, una política global única para enfrentar la gestión de los recursos es un error, puesto que puede desconocer la capacidad de la diversidad natural para crear mejores condiciones para la vida humana.

sociedad y economía • e-ISSN: 2389-9050 • No. 38, 2019 • pp. 6-20
El desarrollo sostenible ya no es un mero ideal de largo plazo. Es un propósito colectivo hacia el cual se encamina la formulación de políticas públicas evolutivas o incrementales, edificadas sobre la relevancia territorial y sobre el reconocimiento de la necesidad de combinar soluciones de mercado, intervenciones estatales y formas organizadas de autogobierno. En breve, "el objetivo ahora debe ser situar la sostenibilidad en el ADN de nuestra sociedad globalmente interconectada" (Ostrom, 2012).

En ese sentido, las políticas públicas necesitan incorporar las nociones de adaptación y evolución que caracterizan a los sistemas socioambientales, y los sistemas de recursos y de gestión que les dan forma. Procesos de decisión traslapados y la redundancia del diseño institucional son convenientes al momento de enfrentar la complejidad de los dilemas de acción colectiva que trae consigo la cuestión minera. La apuesta deliberada consiste en abordar los arreglos microinstitucionales para lograr explicar factores causales, hacer diagnóstico de la situación acción e identificar los principios de reforma. Se trata de un análisis institucionalista metateórico y multimétodo encaminado a microfundamentar los procesos de política pública en la región, en particular en estudios en escenarios de minería. En este sentido, la investigación se aloja en la búsqueda de la síntesis del aprendizaje y de la acumulación de conocimiento, fundadas en el diálogo disciplinar y metodológico.

Con esa apuesta teórica en mente, el trabajo aplicado se ha desarrollado en Antioquia y sus departamentos vecinos, en particular, respecto a las dinámicas del mercado y gobierno de la minería aurífera. Una primera conclusión de las investigaciones iniciales, consiste en reconocer las dificultades que la ausencia o debilidad estatal le imprimen al contexto microsituacional. $\mathrm{Al}$ respecto, Jorge Giraldo (2013) afirma que:

El Estado colombiano, históricamente, se ha desentendido de la regulación de la explotación aurífera y ello ha traído consigo varios intentos por parte de diversos agentes sociales de establecer reglas propias -informales y regionales- de gestión de la eco- 
nomía del oro y, con ella, de la población, sus asentamientos y sus relaciones sociales y políticas (p. 35).

En efecto, el punto de partida de este programa de investigación, que motivó dicha reflexión sobre las ideas en la minería, es reconocer la complejidad de la adversidad social que existe en el territorio. No obstante, las más recientes investigaciones desarrolladas desde el análisis institucionalista y haciendo uso de las herramientas de la economía experimental, permiten tener una mirada renovada de las potencialidades que poseen las comunidades en sus vivencias cotidianas. Es así como en medio de un contexto de procesos de decisión pública carentes de transparencia y de altos niveles de victimización, gracias al accionar de grupos armados ilegales, también es posible identificar altos niveles de confianza moral, confianza grupal y confianza institucional en algunas entidades con presencia permanente en la región (Iglesia Católica, Universidades, Defensoría y Organizaciones Sociales).

Los juegos económicos que se han empleado en estos estudios, también permiten obtener un orden de magnitud de la disposición a la acción colectiva de los participantes, por medio de escenarios que recrean las condiciones de un dilema social. En donde se pone a prueba la capacidad de contribuir a un propósito común, aunque la decisión de no contribuir arroje un beneficio individual mayor. En otras palabras, se analiza la voluntad de los participantes -los jugadores- de hacer jugadas que favorezcan al grupo (a todos los participantes de los juegos) en lugar de aquellas que lo benefician solo a él, incluso en mayor medida que lo obtenido en la jugada grupal. Los resultados de los ejercicios experimentales en municipios mineros (ver Tabla 1) permiten asegurar que:

\begin{abstract}
Frente a los difíciles desafíos de política en escenarios tan complejos como los del Nordeste y Bajo Cauca antioqueños, donde la confianza ha sido tan duramente golpeada por el conflicto y la vulneración sistemática de los derechos de propiedad, los niveles de cooperación se presentan como los más altos. Esta circunstancia debe ser asumida como una conquista social que debe ser puesta al servicio de la reconstrucción de los deteriorados tejidos de confianza en el territorio (Eslava y Zapata, 2014, p. 302).
\end{abstract}

Este tipo de estudios aporta evidencia empírica a las posibilidades de la sociedad solidaria basada en confianza y acción colectiva, aun en contextos adversos como el de la minería aurífera. No se reduce a buenas intenciones: hay condiciones reales sobre las que se puede edificar la barrera a la maldición de los recursos naturales. Esto justifica, además de hacer viable, la propuesta de inclusión de la comunidad como un actor relevante en la relación entre Estado y mercado.

Ahora bien, con el fin de poner en diálogo las disposiciones a confiar y cooperar que muestran los participantes en los ejercicios experimentales con la cuestión ambiental, conviene traer a colación otro estudio acerca de los comportamientos en el departamento de Antioquia:

El 70\% de los encuestados piensa que se debería dar prioridad a la protección del medio ambiente, aún si esto causa un menor crecimiento económico. Las subregiones en las que esta opinión es más alta son Nordes-

\begin{tabular}{ccc}
\hline \multicolumn{2}{c}{ Tabla l. Resultados de ejercicios experimentales } \\
\hline Municipio & Confianza & Disposición a la acción colectiva \\
\hline Cañasgordas, ANT. & $66,7 \%$ & $46,7 \%$ \\
Puerto Libertador, CÓR. & $53,8 \%$ & $53,8 \%$ \\
San Pablo, BOL. & $50,0 \%$ & $45,0 \%$ \\
Segovia, ANT. & $39,1 \%$ & $56,2 \%$ \\
Tarazá, ANT. & $56,2 \%$ & $58,3 \%$ \\
Total & $\mathbf{5 3 , 0 \%}$ & $\mathbf{5 2 , 3} \%$ \\
\hline
\end{tabular}

Fuente: tomado de Eslava (2014). 
te (78\%), Magdalena Medio (75\%), Suroeste (75\%) y Occidente (74\%). Aunque el $71 \%$ de los encuestados afirma confiar en las organizaciones de protección del medio ambiente, el $17 \%$ de los encuestados es miembro de una organización de este tipo, y tan solo el $5 \%$ participa de manera activa (Giraldo, Casas, Méndez y Eslava, 2013, p. 61).

Allí se evidencia un alto nivel de conciencia ambiental. Aquella que puede explicar ideas de reacción frente a la explotación del recurso aurífero, en especial, con el registro más alto en una zona predominantemente minera como el Nordeste; pero la mala noticia consiste en que esa idea no se logra materializar en participación o movilización social. De nuevo, este pensamiento no está matizado por el hecho de que la actividad minera se realice en condiciones de legalidad, informalidad, ancestralidad o ilegalidad. Nada de esto parece importar en un escenario en donde, en el fondo, se discute la premisa de no explotación -por parte de los ciudadanos, como se soportó empíricamente- contra el derecho de explotación que otorga el título a los empresarios o que por la fuerza se toman los ilegales. Si esa idea de rechazo no se trabaja a partir de la ruptura de la dicotomía entre Estado y mercado como variable del desarrollo, la ausencia comunitaria en las decisiones colectivas hará que ese rechazo transite al activismo en torno al sabotaje, que a veces tiene versiones legales como las consultas municipales, pero también tiene versiones violentas.

No obstante, en los municipios mineros donde se realizaron juegos experimentales en el marco del proceso de investigación amplio descrito arriba, ante la pregunta de la encuesta post-juego ¿qué tema requiere mayor intervención en su municipio? Apenas el $1 \%$ de los jugadores responde que el medio ambiente es la prioridad municipal. Vivienda, empleo, vías y salud son los asuntos prioritarios para más del $60 \%$ de la población encuestada. En sintonía con ello, la participación en organizaciones ambientales apenas alcanza un $6 \%$, al tiempo que el $70 \%$ de las modalidades participativas corresponden al trabajo comunitario, vecinal, religioso y deportivo.
Esto lleva directamente a la cuestión por la responsabilidad. Parafraseando a Mark Platts (2012), el deterioro ambiental es algo que la gente le hace a la gente. Quizá las acciones humanas que causan algún daño no expresan directamente conexiones causales deliberadas, ergo, la intencionalidad puede ser matizada, pero el resultado del conjunto de acciones aisladas de esta naturaleza tiene consecuencias sociales (irreversibles en muchos casos). Nuestra cotidianidad sumida en inercias y falsas razones puede convertirse en un arma nociva que amenaza las condiciones de nuestra propia vida.

El desafío consiste en superar la explicación de gente que hace daño a la gente, para incursionar en conceptualizaciones, metodologías y principios de reforma que permitan identificar cómo se las arregla la gente para enfrentar la tendencia al daño colectivo. Al respecto cabe señalar que:

\footnotetext{
Usar una noción de responsabilidad objetiva ayuda a dar sentido a la idea de que los miembros de una sociedad pueden ser responsables por las consecuencias negativas no intencionadas e incluso imprevistas de políticas, instituciones, prácticas y estructuras sociales que esos miembros al menos mantienen, si no es que promueven activamente (Platts, 2012, p. 189).
}

Resulta preciso acudir a la responsabilidad con rostros, a la erradicación de los verbos sin sujeto $\mathrm{y}$ al ejercicio de sentirse interpelado ante el interrogante de uso manido: ¿qué hacer? En el escenario específico de la gobernanza de la minería aurífera es importante profundizar en la relevancia de la vida cotidiana para resolver los dilemas colectivos, y en la necesidad de identificar a los actores locales y extralocales con responsabilidades directas en la valoración y promoción de las experiencias de política pública evolutiva. Aquellas basadas en la creación, uso y acumulación del saber social, en menoscabo de las certezas emanadas del poder político y el conocimiento técnico. De nuevo, Estado y mercado han demostrado dificultades para gobernar el oro, aquellas que solo podrán resolverse 
si incluyen a la comunidad y sus microfundamentos.

\section{Política pública como acción colectiva: categorías analíticas para una gobernanza minera}

La figura propuesta, para abandonar el péndulo que oscila entre lineamientos estatales y contratos mercantiles, es la tríada que concreta la visión de desarrollo, que incluye a la comunidad como tercer actor y a sus arreglos como relevantes. Además, permite reconocer la multiplicidad de interacciones que pueden darse entre los distintos miembros y, de esta manera, entender la gestión de problemas sociales complejos, especialmente, la posibilidad de desplazamientos en los arreglos comunitarios.

Conceptualmente, esos desplazamientos surgen como resultado de intervenciones o decisiones del Estado y del mercado. Esto es lo que se ha llamado efecto crowding out. Un término inicialmente usado para el estudio de la moneda ("la moneda mala desplaza a la buena") pero cuyo escenario de aplicación puede ser ampliado a vastos sectores de comprobación. Al final, para sintetizar hallazgos de indagaciones por la creación, consolidación y desaparición de arreglos colectivos, es posible asegurar que instituciones malas desplazan a las buenas (Ostrom, 2000), (Cárdenas y Carpenter, 2008). De hecho, es posible encontrar desplazamientos entre acciones del Estado sobre mercado o viceversa, así como también se han hallado efectos nocivos, no intencionados, del accionar estatal o mercantil sobre arreglos comunitarios.

A propósito de las implicaciones de la tenaza en mención, vale la pena destacar que el debate público suele enfocarse en la disputa por la eficiencia o por la equidad, esto es, por la generación o distribución de la riqueza. Con lo cual se adentra en dimensiones impersonales de los arreglos sociales. Por el contrario, la tríada busca remarcar esa centralidad de las personas para darles el lugar que merecen. En palabras de Bruni y Zamagni (2007), la pregunta central en este aspecto es:

\begin{abstract}
¿Cómo hacer para que el mercado vuelva a ser un medio que refuerce el vínculo social, a través tanto de la promoción de prácticas de distribución de la riqueza, que se sirven de los mecanismos de mercado para alcanzar ese objetivo, como de la generación de un espacio económico en el que los ciudadanos que libremente eligen el mercado puedan hacer jugar ( $\mathrm{y}$ en consecuencia volver a generar) valores tales como la reciprocidad, la confianza, la simpatía, valores sin los cuales el propio mercado estaría imposibilitado de subsistir por mucho tiempo? (p. 146).
\end{abstract}

La inclusión de la tríada en el análisis y su reivindicación del papel central de la comunidad, no solo ayuda a responder esta pregunta, sino que permite configurar y pensar un escenario de desarrollo distinto. Uno de corte más humano, más integral, de acuerdo con Sen (2000; 2010). De este modo, es posible realizar aportes a "una versión pluralista y multidimensional del mercado y de las organizaciones económicas" (Bruni y Zamagni, 2007, p. 9). Tarea en la cual confluye la discusión de la ciencia económica, que tiende puentes hacia otros saberes, para encontrar convergencia con los aportes de otras ciencias sociales y así poder aproximarse mejor a las realidades humanas de motivaciones, decisiones, y acciones individuales y colectivas. Los ámbitos económico y político están estrechamente relacionados, ya que las condiciones para la creación de riqueza están influenciadas por las decisiones públicas, al tiempo que el erario se alimenta de la dinámica mercantil. Esto permite entender la preferencia por la tríada (Estado, mercado y comunidad), antes que por el dilema (Estado o mercado), bajo el reconocimiento profundo que:

\footnotetext{
No es la economía lo único que mueve a los seres humanos, ese escenario sería un campo de batalla entre seres autómatas e insensibles. Por fortuna, la biografía humana no sólo tiene fines económicos; el amor por el conocimiento, la admiración por la naturaleza, el amor por la pareja, por los amigos, por los hijos, también nos cautiva, pero con mucha más fuerza que el dinero y sus efluentes (Eslava, 2016).
} 
En breve, el tándem mercado-Estado es insuficiente para abordar la complejidad de la gestión colectiva de los recursos naturales no renovables. Como se ha demostrado, los de la minería del oro igualmente. Es preciso volcar la mirada hacia el arreglo microinstitucional; ese en el que tiene lugar la motivación egoísta, pero también las preferencias prosociales (Bowles y Polanía-Reyes, 2012). Los procesos locales de políticas públicas enfrentan la necesidad de reinventarse cada día y, en esa tarea, los atributos y capacidades de las comunidades ofrecen insumos muy valiosos para tomar mejores decisiones y obtener un mayor logro social. Esto tiene una particular relevancia en el escenario minero y la confluencia de actores que contiene.

Así como la economía ha prestado especial atención a los canales físicos del crecimiento económico, las ciencias sociales en general abogan ahora por la importancia de los canales morales del desarrollo (Bruni y Sugden, 2005). En lugar de asumir la búsqueda del precio correcto por medio de intervenciones con subsidios o incentivos, el diagnóstico del análisis permite darle mayor ponderación a criterios alternos de la decisión colectiva. En este sentido, a continuación se expone una suerte de canales civiles del desarrollo que abogan por una gobernanza comunitaria construida desde cimientos prosociales, que contribuyen a procesos de políticas públicas. Estos insumos nutren conceptualmente la propuesta aquí planteada de gobernanza de la minería del oro desde la tríada de Estado, mercado y comunidad.

Aguilar (2013) propone una concepción de gobernanza como proceso de orientación, de guía en la sociedad, que reconoce la existencia de varios actores en el marco de interacción. Lo que refuerza la importancia de la tríada entre Estado, mercado y comunidad. Ese proceso de dirección, denominado gobernanza, convierte preferencias sociales en hechos sociales (Aguilar, 2013). Así los actores definen una situación problemática que detona un proceso intencional, para encaminar acciones colectivas hacia el logro de los objetivos preferidos socialmente.

Las preferencias tienen como característica que se expresan, se argumentan y se acuerdan, buscando que se consoliden o concreten en acciones materiales específicas. De este modo, la gobernanza es el proceso que orienta el debate y la acción, que lleva el acuerdo a la concreción de acciones.

Las políticas públicas, en un escenario de gobernanza entendido de esta manera, no pueden tomarse como los productos de la elaboración técnica exclusiva del ejecutivo, sino como un proceso de acercamiento entre los actores de la tríada, como el resultado de interacciones, de acción colectiva. Y como tal incluyen varios actores, en distintos niveles y escenarios de actuación comunitarios y gubernamentales.

Esto permite avanzar un paso desde el planteamiento de Alejo Vargas de política pública entendida como el "Conjunto de sucesivas iniciativas, decisiones y acciones del régimen político frente a situaciones socialmente problemáticas y que buscan la resolución de las mismas o llevarlas a niveles manejables" (Vargas, 1999, p. 29). Hacia una concepción más amplia, que no desmerita el planteamiento inicial, sino que lo retoma y complementa, donde la variable comunitaria entra al juego de la decisión. Ya no como consulta, sino como actor central.

Joan Subirats (2010), por su parte, señala que una política pública necesita de una definición "operativa" que muestre la distancia entre la situación problemática de partida no deseada y la situación que, sin ser la "óptima (el no problema)", sea mejor que la de inicio. Según su planteamiento:

El problema estriba en definir e impulsar políticas y medidas que vayan en el sentido deseado y que congreguen el máximo consenso social posible, ampliando los individuos y grupos sociales conscientes del problema, y reduciendo y restringiendo la capacidad de maniobra y de alianza de los actores que tratan de mantener el status quo (Subirats, 2010, p. 5).

Lo anterior, sin desconocer que se ha entendido, en el ámbito académico, que las políticas públicas tienen un carácter bidimensional entre lo técnico y lo político (Subirats, 2010), (Eslava, 2011; 2017), 
(Vargas, 1999). Lo cual no contradice la tríada propuesta, en la medida en que la vivencia comunitaria complementa las consideraciones técnicas y políticas, no las confronta. Al menos no por definición. No obstante lo anterior, ya se mencionaba más arriba la siempre presente posibilidad de desplazamiento que los actores en la tríada generan con sus acciones sobre los demás actores, en especial el Estado y el mercado sobre la comunidad. Las políticas públicas no son lejanas a este postulado y en tal sentido pueden causar daño a las comunidades que pretenden beneficiar (Eslava, 2017). También pueden verse nutridas de compromisos colectivos que encierran objetivos distintos de lo que aquí se llama acción colectiva prosocial, es decir, pueden verse nutridas por acciones de cooperación que se consideren con fines negativos. Lo que Robert Putnam (2002) describe como el lado oscuro del capital social.

El apego a macrohipótesis para la orientación de la sociedad trae consigo la posibilidad del daño a los públicos que se pretende beneficiar. De acuerdo con Ostrom (2013) y la evidencia experimental de sus estudios: "La regulación externamente impuesta que llevaría a retornos conjuntos más altos, en realidad "desplazaba" las conductas voluntarias tendientes a la cooperación" (p. 35). Sin embargo, ese efecto puede contrarrestarse comprendiendo los rasgos comunitarios que configuran el insumo prosocial de las políticas o políticas públicas como acción colectiva. El reto consiste en evitar el crowding out (efecto desplazamiento), previo trabajo de valoración de la magnitud de los atributos y capacidades de comunidad en juego.

Contrarrestar el efecto -o concretar un crowding in- parte de reconocer que en un escenario de política pública, como acción colectiva, el poder de la política, el saber técnico y la vivencia comunitaria no solo no riñen en cuanto a gobernabilidad, sino que pueden y encuentran en la realidad mecanismos de cooperación y complementariedad (Bowles, 2016).

La gobernanza es política pública desarrollada desde la acción colectiva. Lo que lleva a evidenciar la presencia de rasgos de comunidad que pueden caracterizarse como atributos y disposiciones co- lectivos, que están presentes y que son identificables como el activo de la comunidad en la tríada y su contribución al posible desarrollo de política pública en su vertiente prosocial. Si antes se mencionó que la visión dicotómica dejaba por fuera a la comunidad de la interacción entre Estado y mercado, otro efecto de dicha exclusión es la invisibilización de los procesos de política pública en su variante de acción colectiva, vistos únicamente como resultado del ejercicio del gobierno. Al final lo que se busca es que la mano invisible del mercado competitivo y el puño del Estado planificador, se vean complementados con el apretón de manos de la confianza en la comunidad. Esto lleva a que una sociedad sea gobernada bajo mejores arreglos colectivos (Bowles y Gintis, 1998). De acuerdo con Ostrom (2013):

\begin{abstract}
Las hipótesis clásicas -que sostienen que los individuos racionales se enfrentan a una bifurcación entre formas organizativas y bienes- ocultan los esfuerzos potencialmente productivos de individuos y grupos para organizar y resolver dilemas sociales, tales como la sobreexplotación de recursos comunes y la falta de provisión de bienes públicos locales (p. 20).
\end{abstract}

Pocos recursos como los naturales (en particular el oro) caben de manera tan perfecta en la definición de recursos comunes. De ahí que se parta del reconocimiento de la existencia de la tríada y su relevancia, además de la hipótesis que entiende la gobernanza como proceso orientador que sintetiza y alberga la política pública como resultado de la acción colectiva. La gobernanza de la minería del oro busca justamente eso. El mecanismo de conexión teórica entre gobernanza, política pública y acción colectiva, se rastrea en los atributos de comunidad prosociales, que pueden ser informales. Ese es el foco de la tarea aquí propuesta para la gobernanza de la minería del oro: indagar por los atributos de comunidad y arreglos comunitarios que favorecen la acción colectiva que genera políticas públicas, es decir, que son prosociales. En palabras de Ostrom (2013): "Atributos de una comunidad, que pueden incluir la historia de interacciones previas, homogeneidad o heterogeneidad interna de atributos clave, y el conocimiento y capital social de aquellos que pueden participar 
o verse afectados por otros" (p. 17).

Para ello, se parte de la premisa según la cual el diseño institucional es pieza clave del cambio social. Por ejemplo, el diseño de políticas públicas puede ser el primer paso para detonar cambios en las realidades adversas que enfrentan los públicos de tales políticas. En principio, no hace falta abogar por grandes reformas en los procesos de generación y acumulación de capitales, pues un pequeño empujón, en la dirección correcta, permite a las personas tomar mejores decisiones individuales, con saldo positivo para el colectivo (Sunstein y Thaler, 2017), (Sunstein, 2017).

Las motivaciones humanas van más allá de la consideración monetaria y las lógicas de elección racional pura; dan cabida a coincidencias entre acción individual y acción colectiva. Las macrohipótesis de gobernanza y acción colectiva se deben complementar con visiones más locales de los microfundamentos, acerca de lo que las personas tienen en mente a la hora de decidir e interactuar. De allí la relevancia de volver a lo que las comunidades piensan sobre la minería del oro y su explotación, y a los microfundamentos de los escenarios mineros, para entender la manera en que pueden y deben acercarse a la institucionalidad de los municipios, los mineros y las comunidades. En pro del desarrollo y la generación de políticas públicas en clave de gobernanza de la minería.

En breve, las dinámicas locales exhiben comportamientos prosociales que se evidencian en disposiciones a confiar y a cooperar incluso con desconocidos. El reto consiste en construir el puente que conecte a este activo comunitario con los procesos de política pública local. A ese propósito se encaminan los principios de reforma sintetizados en la Tabla 2 y que ayudan como preludio para las consideraciones finales.

\section{Consideraciones finales}

El amplio consenso de los tomadores de decisiones en el país alrededor del modelo extractivista hace que las políticas de alcance nacional se soporten en ideas limitadas, que conciben el recurso natural (en este caso el oro) como insumo intermedio o materia prima. De la explotación de ese recurso se deriva una renta estatal a manera de compensación, que

Tabla 2. Principios de reforma

1. Políticas públicas basadas en gobernanza comunitaria. En contraposición a la ingeniería social de la política pública, según la cual los resultados sociales son determinados por las acciones autónomas de funcionarios motivados por lo público, la gobernanza comunitaria aboga por una interacción compleja entre información, distribución de riqueza, conflicto y preferencias.

2. Construir instituciones locales ingeniosas. Las personas están dispuestas a invertir tiempo y energía en el diseño y adaptación de reglas que les permitan obtener resultados colectivos.

3. Encarar los problemas de pobreza, exclusión y ambientales. La intervención estatal en las zonas mineras debe estar dirigida a establecer el imperio de la ley y la regulación de las actividades económicas y sociales, pero si no se atienden inmediatamente las necesidades de los más pobres -que lo han sido secularmente- el Estado aparecerá ante la población como una institución extractiva más.

4. Redundancia en la creación de normas. Así como los gobiernos deciden invertir cuantiosos recursos en la difusión repetitiva de ejecutorias, el proceso de diseño institucional también debe incursionar en la práctica de la reiteración. Es decir, además de la regla escrita en Acuerdos, Ordenanzas, Decretos o Leyes, también son importantes los acuerdos tácitos y simbólicos que, de la mano del despliegue mediático, refuercen la idea central de la regla.

5. Tener en cuenta los comportamientos prosociales. Es conveniente reconocer y promover los comportamientos cotidianos que construyen comunidad: la confianza en el otro y la disposición a emprender acciones comunes constituyen el par necesario para dar forma a la noción de un nosotros.

6. Avanzar en la formalización, pero sobre todo en la construcción de nexos fuertes entre sector formal e informal. Las políticas que incentiven la formalización de la actividad económica minera deben revisarse en busca de una mayor eficacia.

Fuente: tomado de Eslava (2014). 
incrementa el recaudo fiscal para hacer política social redistributiva. Este proceso se hace sin considerar el estado en que quedan los territorios una vez que la renta natural no renovable se agota $y$, con ello, la tributación compensatoria y las demás actividades económicas que terminan.

Ahora bien, la presencia de soluciones desde el predominio del mercado o del Estado puede incurrir en el error de causar daño a los arreglos colectivos que existen en un territorio. Es lo que aquí se ha presentado como crowding out o daño a la acción colectiva.

Es importante darle cabida a una discusión que permita superar la fe convencional en el mercado y el Estado para darle importancia a la comunidad. La lógica de mercado agota el subsistema de recursos y la precariedad estatal permite un subsistema social de gestión elitista. Las buenas noticias provienen del arreglo comunitario, de sus acuerdos para evitar la sobreexplotación y de su diseño institucional para ponerse reglas y cumplirlas.

Por supuesto, el mercado debe abordarse desde las cuestiones por el modo de producción, comercialización y consumo, así como la intervención estatal es indispensable para fijar restricciones y posibilidades, pero la cuestión ambiental también exige poner atención en la vida cotidiana de la comunidad. El diagnóstico y búsqueda de soluciones no se reduce a la política económica, pues también las reglas informales superan las predicciones sujetas al efecto precio de las distorsiones, y da cabida a las preferencias prosociales, que pasan por alto las consideraciones del análisis costo-beneficio.

La propuesta teórica del artículo se puede sintetizar en torno a la gobernanza comunitaria de la minería del oro. La cual aboga por la construcción de arreglos colectivos que le den cabida a la tríada de actores compuesta por intervención estatal y mercantil, al tiempo que se identifican y valoran los insumos provenientes de arreglos microinstitucionales de arraigo comunitario. En este sentido, se propone la comprensión de los procesos de política pública como el resultado de acciones colectivas prosociales, esto es, escenarios de deci- sión en los que es posible construir un nosotros para actuar en consecuencia.

La problemática local puede encontrar escenarios de resolución en la economía política de la periferia. En la comprensión e identificación de principios de reforma (dinámica de élites regionales alrededor de la actividad política y económica) para avanzar desde el elitismo prevaleciente, hacia un elitismo distributivo en principio y hacia un marco más plural de gobernanza comunitaria. Es posible concluir, retomando la afirmación de Anthony Bebbington (2013), "la mejora de la regulación necesita de mecanismos que ayuden a transformar el conflicto social en innovación institucional, ya que esta transformación no ocurre de manera automática" (p. 27).

Luego de muchas décadas de historia extractiva hay dos actores que permanecen en relaciones más o menos inalteradas en los territorios: un Estado que permite la extracción del recurso natural no renovable por parte de un privado que por ello hace cierta compensación tributaria. Los resultados han sido básicamente los mismos: territorios de gobernanza en disputa, con presencia de actores ilegales que son predadores de la renta natural en un contexto generalizado de pobreza, informalidad y necesidades básicas insatisfechas. En toda esta historia hay un actor que se ha quedado al margen y unas relaciones que, como atributos de ese actor, están por explorar: la comunidad y sus atributos prosociales de confianza y disposición a la acción colectiva. Abrir el juego a su participación permite esperar resultados distintos a los ya conocidos.

La tarea concreta consiste en superar el pesimismo estéril y construir alternativas viables. Esa apuesta está ubicada en el terreno de las decisiones políticas que se toman en un territorio: fortalecer nuevos liderazgos y acompañar desde fuera para brindar el blindaje ante las resistencias. En ese caso, es vital el papel del sector privado, del sector social y de otras entidades con presencia en las regiones a través de acciones de responsabilidad social, movilización social y transparencia de los procesos de decisión. 
Ideas sobre la minería en Antioquia. Desarrollo, gobernanza comunitaria y arreglos colectivos

\section{Referencias bibliográficas}

Aguilar, L. F. (2013). Gobierno y administración pública. México D.F., México: Fondo de Cultura Económica.

Basu, K. (2013). Más allá de la mano invisible. Fundamentos para una nueva economía. Ciudad de México, México: FCE.

Bebbington, A. (2013). Industrias extractivas. Conflicto social y dinámicas institucionales en la Región Andina. Lima, Perú: IEP - CEPES - Grupo Propuesta Ciudadana.

Bowles, S. (2016). The Moral Economy. New Haven, US: Yale.

Bowles, S. y Gintis, H. (1998). Efficient redistribution: New rules for markets, states and communities. Recuperado de http://www.umass.edu/preferen/gintis/efficient.pdf

Bowles, S. y Gintis, H. (2005). Social Capital, Moral Sentiments, and Community Governance. En H. Gintis, S. Bowles, R. Boyd y E. Fehr, Moral Sentiments and Material Interests. The Foundations of Cooperation in Economic Life (pp. 379-398). Cambridge, US: MIT.

Bowles, S. y Polanía, S. (2012). Economic Incentives and Social Preferences: Substitutes or Complements? Journal of Economic Literature, 50(2), 368-425. http://doi.org/10.1257/jel.50.2.368

Bruni, L. y Sugden, R. (2005). Los canales morales: la confianza y el capital social en la obra de Hume, Smith y Genovesi. Revista Valores en la Sociedad Industrial, (62), 51-70.

Bruni, L. y Zamagni, S. (2007). Economía civil. Eficiencia, equidad, felicidad pública. Buenos Aires, Argentina: Prometeo Libros.

Burchardt, H. (2014). Logros y contradicciones del extractivismo. Bases para una fundamentación empírica y analítica. Buenos Aires, Argentina: Nueva Sociedad.

Cárdenas, J. C. (2009). Dilemas de lo colectivo. Instituciones, pobreza y cooperación en el manejo local de los recursos de uso común. Bogotá, Colombia: Uniandes.

Cárdenas, J. C. y Carpenter, J. (2008). Behavioural development economics: Lessons from field labs in the developing world. The Journal of Development Studies, 44(3),311-338. https://doi.org/10.1080/00220380701848327

Eslava, A. (2011). El juego de las políticas públicas. Reglas y decisiones sociales. Medellín, Colombia: Fondo Editorial Universidad EAFIT.

Eslava, A. (2014). Oro como fortuna. Instituciones, capital social y gobernanza de la minería aurífera colombiana. Medellín, Colombia: Universidad EAFIT - Colciencias.

Eslava, A. (2016). Una visión amplia del desarrollo. Apuntes de Economía Política. Recuperado de https:// www.researchgate.net/publication/308972261_UNA_VISION_AMPLIA_DEL_DESARROLLO_Apuntes_de_ Economia_Politica_Version_preliminar-161001-

Eslava, A. (2017). Políticos, técnicos y comunidades. Una visión institucionalista del urbanismo social en Medellín. Medellín, Colombia: Fondo Editorial Universidad EAFIT.

Eslava, A. y Zapata, O. (2014). Confianza y acción colectiva en contextos mineros: un análisis econométrico. En A. Eslava (Ed.), Oro como fortuna (pp. 287-315). Medellín, Colombia: EAFIT - Colciencias.

Garay, L. J. (2013). Minería en Colombia. Fundamentos para superar el modelo extractivista. Bogotá, Colombia: Contraloría General de la República.

Garay, L. J. (2014). Una alternativa al modelo neo-extractivista. Conferencia llevada a cabo en Cambio Climático, Economía y Políticas Económicas. FESCOL - FNA - Plataforma Climática Latinoamericana, Bogotá, Colombia, 28 de mayo de 20114 . 
Giraldo, J. (2013). El gobierno del oro en el bajo Cauca. En J. Giraldo (Ed.), Economía criminal y poder político (pp. 33-68). Medellín, Colombia: EAFIT.

Giraldo, J., Casas, A., Méndez, N. y Eslava, A. (2013). Valores, representaciones y capital social en Antioquia. Medellín, Colombia: EAFIT - SURA - Gobernación de Antioquia.

González, J. I. (2003). Economía y ética. Bogotá, Colombia: Universidad Externado de Colombia.

Guha-Khasnobis, B., Kanbur, R. y Ostrom, E. (2006). Beyond Formality and Informality. En B. Guha-Khasnobis, R. Kanbur y E. Ostrom, Linking the Formal and Informal Economy: Concepts and Policies (pp. 1-18). Oxford, UK: Oxford University.

Ostrom, E. (2000). Crowding out Citizenship. Scandinavian Political Studies, 23(1), 3-15. https://doi. org/10.1111/1467-9477.00028

Ostrom, E. (2012). Green from the Grassroots. Recuperado de http://www.project-syndicate.org/commentary/ green-from-the-grassroots

Ostrom, E. (2013). Más allá de los mercados y los Estados: Gobernanza policéntrica de sistemas económicos complejos. Revista de Derecho Ambiental de la Universidad de Palermo, (1), 5-72.

Platts, M. (2012). Ser responsable. Exploraciones filosóficas. Ciudad de México, México: UNAM.

Poteete, A., Janssen, M. y Ostrom, E. (2012). Trabajar juntos. Acción colectiva, bienes comunes y múltiples métodos en la práctica. Ciudad de México, México: FCE.

Putnam, R. (2002). Solo en la bolera. Barcelona, España: Galaxia Gutenberg.

Sen, A. (2000). Desarrollo y libertad. Bogotá, Colombia: Planeta.

Sen, A. (2010). La idea de la justicia. Madrid, España: Taurus.

Suárez, A. (2013). La minería colonial del siglo XXI. Bogotá, Colombia: Aurora.

Subirats, J. (2010). Políticas públicas e inclusión social. Factores territoriales y gobiernos locales. Recuperado de https://www.academia.edu/351253/Pol\%C3\%ADticas_publicas_e_inclusi\%C3\%B3n_social

Sunstein, C. (2017). Paternalismo libertario. Barcelona, España: Herder.

Sunstein, C. y Thaler, R. (2017). Un pequeño empujón. El impulso que necesitas para tomar mejores decisiones sobre salud, dinero y felicidad. México D.F., México: Taurus.

Vargas, A. (1999). Notas sobre el Estado y las políticas públicas. Bogotá, Colombia: Almudena Editores. 